can comprehend only very imperfectly, and that must fill a thinking person with a feeling of humility"' (pp 39 and 40) are two such passages. But there are many, and even if one does not fully agree with them, all are refreshing. He also defended philosophy in general against its deprecators even when recognising its weakness: "Philosophy is like a mother who gave birth and endowed all other sciences" (p106) is perhaps an exaggeration but a well-meant one.

Einstein's love of poesie is also little recognised. He even wrote some poems, all of a slightly humorous nature, for his letters. About ten of these are contained in the book, and all are amusing.

Einstein's attitude towards religion is also clearly expressed in his letters. He felt - as many people do today - that its main role should be in the ethical domain, whereas its cognitive assertions should be more in the background. He also writes a good deal about the extinction of life - one must, with sadness, come to the conclusion that he enjoyed his last few years very little and considered the extinction of his life not only cognitively, but to some degree also emotionally, as a liberation from obligations.

Einstein's political convictions are also very apparent from his letters. These are, of course, generally known: he was opposed to all dictatorships, be they of the national socialistic - that is, Hitlerinspired and anti-semitic - or of communistic nature. In fact, one draft letter (pp 110-112) is so vehement that it was not sent out but, according to the introduction preceding it, "must have brought him a feeling of relief"'.

The preceding discussion contains, of course, only a superficial review of the kind of information the reader will gain about Einstein's interests and thinking when reading this book. And the information will appear in a much more lively fashion than the foregoing summary presents it.

The book contains more than a hundred letters. To whom were they addressed? A few to his sister, Maja, a few more to his earliest physicist friends Zangger, Ehrenfest and Lanczos, but even more, about eight, to his psychiatrist friend Juliusburger. There is no letter to either of his wives or to the rest of his family. The rest of the letters are practically all answers to letters he received, each from a different person. With about half of these he had some acquaintance, the other's being written to please or comfort people he had not heard of before. Many of these letters, the writing of which was stimulated by kindness or compassion, contain passages which are truly memorable, and manifest deep insights. A few of the other letters are directed to editors of journals and about four to ministers of churches. These latter all have a strong political undertone.

It is not clear how the letters published were selected, nor is it clear how Einstein had chosen the letters to be answered. Ir one of his letters (p43) he praises the one which he answers as being very reasonable, in contrast to the hundreds of other letters which he was receiving, most of which, of course, he did not answer. It would also be interesting to know where and how the letters and the drafts of the letters published were found.

The English language part of the book (the first 115 pages) are arranged into 12 sections. These sections, however, have no titles and it is not clear on what basis the various letters are distributed into these sections. This has advantages if one reads the book from cover to cover - it is not easy to digest 10 philosophical letters in succession and not refreshing to read several complaints about politicians' misdeeds one after the other. But if one wants to recover a passage which one remembers vaguely, it would be good to have either a definite order of the articles in the book, or at least an index at the end of it. Similarly, the time ordering of the letters seems to be a

\section{The quantum and its supporting
planks}

\section{P.T. Landsberg}

Black-Body Theory and the Quantum Discontinuity, 1894-1912. By T.S. Kuhn. Pp. 512. (Clarendon/Oxford University Press: Oxford and New York, 1978.) $£ 12$.

THE realisation that discontinuities (or quanta of energy) are needed for the description of microscopic phenomena represented one of the great transformations of human thought. Not that everything was believed to be continuous before that - the atomic concept of Democritus, the distinct notes of musical instruments, comets and explosions had been part of the intellectual furniture for centuries, and they certainly implied discontinuities of sorts. But they were not fundamental. Comets continued to be, when not in view; explosions could presumably be analysed into a more or less continuous sequence of chemical reactions; musical notes could be changed by altering the tension in the wire; and the constitution of atoms (if they existed, one would add in 1900) posed in any case intractable problems, for example, how could they be indivisible if they participated in the production of spectral lines? Indeed, as W.K. Clifford observed, an atom must be at least as complicated as a grand piano. Upon this placid scene, in which physics seemed to be approaching a completed structure, there floated gently (for it did not 'burst') Planck's theory of random one. Thus, Section 6 (pp54-74) has articles dating from ' 33 , then ' 51 and ' 53 , then '27, again ' 33, '35, '21, and so on, ending up with one of '27. The subjects vary equally greatly, the first is political, the next a thank-you for the offer of financial help, the next refers to physics, then comes a letter to a student publication, then a praise of science in general and so it goes on. It is amusing to read all this, but a catalogue surely would be useful.

After all this criticism, let me compliment again the Selectors and Editors for the very good job they have done. It surely required not only knowledge but also devotion to the job and a true interest in the human side of Einstein.

Eugene Wigner first met Einstein in 1925, while a student in Berlin. He had closer contact with him after 1933 when both lived in Princeton, New Jersey.

the energy density, as a function of frequency, of radiation when in thermal equilibrium with bodies at temperature $T$ (black-body radiation). Shortly afterwards arose the problems of relativity, specific heat anomolies, etc., and so black-body radiation never lost the charisma of having been the first problem area which brought deep changes to classical physics. It did so by suggesting that an atom oscillating linearly at a frequency $v$ has available only discrete energies $h v, 2 h v, \ldots$ and so on, whereas classical theory would have allowed a continuous range of energies. Here $h$ is the newly introduced constant of nature, now called Planck's constant.

At precisely what time and through whose pen did these discontinuities enter physics? The normal answer is that the year was 1900 and the man was Max Planck. In this scholarly study, which can boast 90 pages of notes and references, Kuhn suggests an amendment. Planck restricted the energy of $N$ oscillators to be integral multiples of an energy unit, $\varepsilon$ say, a type of "quantisation" already envisaged by Boltzmann in 1877, but he did not impose this constraint on a single oscillator until after 1906 (see p126), the year in which this requirement had been emphasised independently by Paul Ehrenfest and Einstein, "the two physicists who first recognised that Planck's black-body law could not be derived without restricting resonator energy to integral multiples of $h$ or some equivalent non-classical step" (page ix).

In the Epilogue Kuhn discusses Planck's "second theory" of heat radiation (1911) which introduced the notion that the mean energy of an atomic oscillator goes to a constant and non-zero value at the lowest temperatures. Although this zero-point energy remained important, Planck's second theory was 
also to suffer profound amendments. By now, as Kuhn shows, the scientific community was turning to the quantum theory of specific heats.

Scientific community? In those far off days of the first Davis Cup matches, of Henry Ford and Thomas Mann, the number of authors who wrote on blackbody theory did not exceed 20 even by 1914. By that time there were still only about sixty-five authors who wrote on the quantum theory, but the number was rapidly rising (as clear from the only three figures in the book). Does today's science harbour another growth point of this significance so that mathematically simple but conceptually gigantic leaps can again be made? Those who doubt that such a growth point can still be found in physics hope, like drowning men clutching at a straw, that it is perhaps still possible in biology. It is, however, a delight of science that there could yet be hope for physics. Who knows?

Mathematics is not popular with readers of book reviews, but to understand the background of Planck's arguments a gentle sniff of three results is needed. First, as black-body radiation does not depend on the material systems with which it is in contact (Kirchhoff, 1860), the simplest systems can be chosen. These are oscillators, $N$ in number say for each frequency $v$. The mean energy $U(v, T)$ of one of them is then simply related to the energy density $e(v, T)$ of the radiation (Planck, 1899). Secondly, thermodynamics tells us how to find the mean entropy $S(U)$ of an oscillator in terms of $U$, provided $U(v, T)$ is known. (Example: if $U=k T$, then $\mathrm{d} S / \mathrm{d} U=1 / T=k / U$, so $S=k \ln ()$. Thirdly, if one has a fundamental model to calculate the number $W$ of so-called microstates of a system one can evaluate the entropy with the aid of a simple relation (Boltzmann 1877).

Now a remark about three Planck papers. In March 1900 he injected an empirical formula for $e$ due to Wien (1896) into this theoretical structure, and went from $Q$ to $U$ to $S$. But then he could only check certain properties of $S$ and nothing revolutionary resulted.. In October 1900 he injected into the same structure a new $(S, U)$-relationship which contained the classical result $\mathrm{d}^{2} S / \mathrm{d} U^{2}=-k / U^{2}$ and the Wien result $\mathrm{d}^{2} S / \mathrm{d} U^{2}=-A / U$ as limits. It was a trial, or interpolation formula, and, passing in the opposite direction now, he inferred $U$ and $Q$. The latter result was what has since become known as the famous Planck distribution law. "Permit me to direct your attention to this new formula" says Planck, "... I consider it to be the simplest apart from Wien's." But 1 had no firm basis in theory! Planck tells us that he worked very hard to find such a basis and on 14 December 1900, he produced a $W$-expression from the mechanics of oscillators. This is where he was very brief and not very clear. In any case, he now proceeded in the accepted logical direction from $W$ to $S$ to $U$ and $Q$, and that was the essential job done. Or was it?

The problem for the studious reader, 75 years later, is: how did he get $W$ ? He says about the total energy of the $\mathrm{N}$ oscillators (we suppress the suffix $\nu$, as we consider a given frequency), that "it is necessary" that it shall be considered to be "not a continuous, infinitely divisible quantity", but that "it shall be considered to be discrete and to consist of a finite number of equal magnitudes" (Ann.Phys. $\dot{2}$, 4,553; January, 1901), "which is the most essential point of the whole calculation" (Verh.d.Dtsch.Phys, Ges., 2, 237; December, 1900).

Kuhn unfortunately does not quote these passages, and argues that the fact that the total $\mathrm{N}$-oscillator energy $E_{N}$ is an integer $n$ multiplied by an energy unit $\varepsilon$ does not give the essence of the discontinuity: "If quantization is the subdivision of total energy into finite parts, then Boltzmmann is its author" (p127). this is a possible point of view. An alternative, more generous, interpretation is to recall, first, that the oscillators are assumed far enough apart not to interact with each other. Hence, if one adds a single oscillator, then its average energy $E_{1}=U$ must be the difference between the two Planckian results $E_{N+1}-E_{N}=n \varepsilon-m \varepsilon$ where $n$ and $m$ are integers. Hence Planck's result extends to $U=r \varepsilon(r=1,2, \ldots)$. Secondly, what was still a fictitious model for Boltzmann, who after all took the limits $\varepsilon \rightarrow N \rightarrow \infty$, had become physically relevant in Planck's hands. Nevertheless, it is certainly true that Planck's reticence is puzzling, as has been pointed out (for example, by M.J. Klein, Paul Ehrenfest, Vol I, p228, North-Holland: Amsterdam, 1970; by H.-G. Sch ठpf, Von Kirchoff bis Planck, p106, Akademie: Berlin, 1978). Although there will therefore be discussions among historians of science sparked off by this book, we shall never know exactly what was in Planck's mind in 1900, nor is it very important to know. It strikes me that Planck knew the chain $Q-U-S-W$ very well and had great confidence in his $\varrho$; so he worked backwards to find the $W$ needed. Perhaps it was because Boltzmann had done much of the mathematics needed by Planck that Planck did not expound the theory of the $W$ as clearly as he might have done. That there existed a sensible expression at all was to him, and rightly, an achievement. To infer single oscillator quantisation (which he might have done by the argument given above) was in 1900 against his wish, as he did not want to leave classical physics, as Kuhn reminds us again so clearly. In any case should an average oscillator energy be quantised? Surely not, a point apparently missed by some later commentators.

As to Einstein, he emerges as hardly ever wrong, as the following story shows. On 12 June 1913, Planck, Nernst,

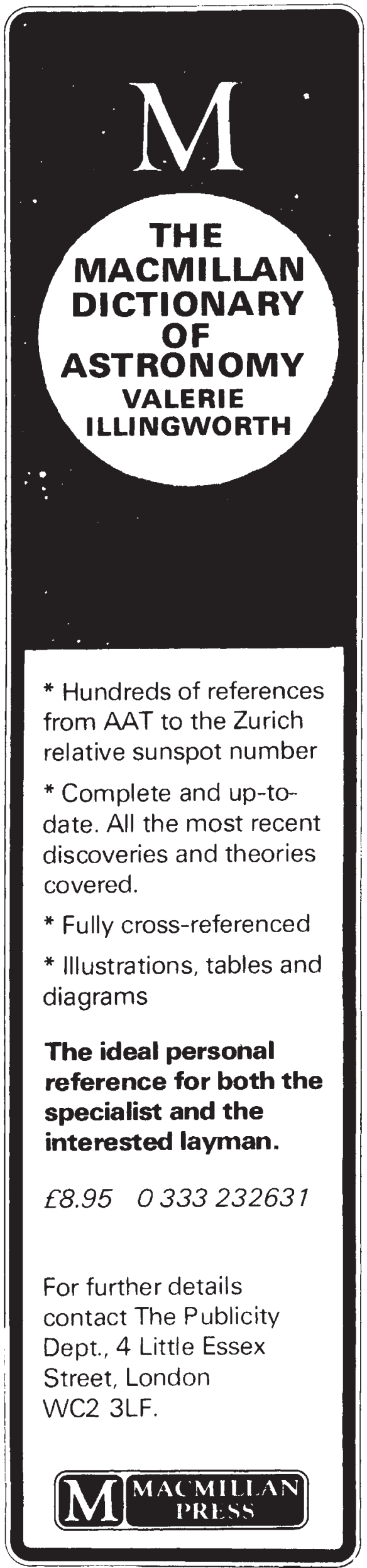

Circle No. 74 on Reader Enquiry Card. 
answer, as to whether systems other than oscillators, for example rotators, were studied in contact with black-body radiation. If not, why not? In any case, the element of confusion between $E_{N}$ and $E_{1}$ and between quantising $E_{1}=U$ as against quantising the unaveraged single oscillator energy, means that the Planck argument has to be replaced. By treating radiation as a gas of "light quanta" and introducing the notion of indistinguishability of quanta of given energy, Bose (1924) gave an argument which no longer depended on any parameters of the material system in contact with the radiation. In a modern exposition Bose's $W$ had the same form as Planck's, $W=(N+P-1) ! / P !(N-1) !$. However, Planck oscillator number $N$ became the number of states in a typical

Sorry, for copyright reasons some images on this page may not be available online

Rubens and Warburg proposed Einstein for full membership of the Prussian Academy of Sciences, describing the achievements of the 34-year-old scientist in glowing terms. From the point of view of the book under review it is of interest that they did, however, remark: "That he has occasionally missed the target in his speculations, as for instance in his light quantum hypothesis, is not something that should count too severely against him; for even in the most exact of sciences it is not possible to introduce real advances without occasionally taking a risk". One can almost hear Planck's voice. It is a sobering thought for those called upon to judge the work of their peers in scientific academies, research committees, book reviews and promotion boards that this distinguished group of four was so devastatingly wrong; after all the light quantum hypothesis was essential to Einstein's explanation of the photoelectric effect which was to be cited by the Nobel Committee in 1921. This interesting matter did not escape Kuhn's eagle eye (though he attributes it to 1914) but he notes it only in passing ( $p 182$ ). Yet there it is. The careful and conservative Planck held back. Friend and supporter of Einstein's though he was - to the extent of risking the concentration camp by his 1933 declaration in support of Einstein the photon picture of radiation was too much in advance of current thinking in 1905.

My main criticism of this fine book - so lovingly researched as to include abstracts, reviews and relevant citations of some of the key papers - is that this very detail has prevented a tight organisation of the material and of the author's conclusions, which are therefore somewhat buried at various points, in spite of a good index. The Epilogue might have included a two- or three-page forward look into the 1920 s, for reasons which will be touched on below.

An alert reader may ask a question, to which the book does not supply an narrow energy range of the quanta considered, and Planck's integer $\mathrm{P}=E_{N} / \varepsilon$ became the number of quanta to be distributed over these states in an optimal manner. (For Planck $\boldsymbol{P}$ was the number of discrete energy elements to be distributed over the $N$ oscillators). Although this was much more satisfactory, the result was approximate (Stirling's approximation). On the whole this does not worry people. However, one cannot conceal that this approximation breaks down for the small number of quanta which can be found in high energies, and I found (1954) that if the Stirling approximation is improved one gets into trouble. The Planck law can be derived as an exact result, but as far as I know only by using the grand canonical ensemble or by taking a limit.

Two queries may be in order in conclusion. In "overblowing" a wind instrument the note jumps as a higher mode is excited. This is typical of classical discontinuities. They are not mentioned in the book but it would be of interest to study them, together with Schrodinger's and Planck's approaches, in a historical context. Secondly, the case studied here suggests (though one must not generalise from one example) that a great scientific advance - a revolution if you like - tends to dissolve into little steps on closer inspection. The same man may take different steps at different times, so that he may in the end have made a major contribution; but small steps they are nevertheless. If this is a general rule, with a few exceptions such as general relativity, it would be interesting to hear Kuhn, the author of the Structure of Scientific Revolutions (1962) consider it in the light of this latest book. The topic itself has been much discussed of course (for example, by S. Toulmin, Human Understanding. Vol. 1, 1972), but it is particularly interesting in connection with the story of black-body radiation.

P.T. Landsberg is Professor of Mathematics at the University of Southampton, UK. 